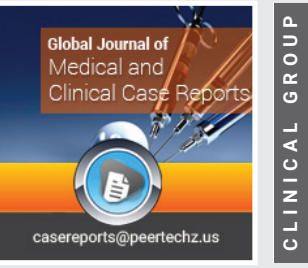

\section{Post traumatic facial artery pseudoaneurysm-A case report}

\section{Raisa Altaf, Imtiaz Ali*, Rabiya Siraj, Jawaid Iqbal and Faiza Hassan}

Department of Radiology, Liaquat National Hospital and Medical College, Karachi, Sindh, Pakistan
Received: 27 December, 2021

Accepted: 03 January, 2022

Published: 04 January, 2022

*Corresponding author: Imtiaz Ali, Senior Registrar, Department of Radiology, Liaquat National Hospital and Medical College, Karachi, Sindh, Pakistan, Tel: +923337071939; E-mail: imtiazpanhwer17@gmail.com

Copyright License: (c) 2022 Altaf R, et al. This is an open-access article distributed under the terms of the Creative Commons Attribution License, which permits unrestricted use, distribution, and reproduction in any medium, provided the original author and source are credited.

https://www.peertechzpublications.com

\title{
Abstract
}

Facial artery a branch of external carotid artery rarely develops an aneurysm due to its superficial location and small size that mostly leads to complete dissection rather than laceration of the vessel. Pseudo-aneurysms develop in the superficial temporal arteries in this region. Rupture of this aneurysm can lead to extravasation of blood within the adjacent soft tissues resulting in hemorrhage in some cases. Only a few cases of facial artery aneurysms have been reported in publications. We are therefore discussing here a case of left facial artery pseudoaneurysm secondary to trauma and its radiological imaging findings on CT angiogram.

\section{Introduction}

Facial artery also known as the external maxillary artery, is a branch of external carotid artery that arises near the angle of the mandible and supplies the muscles of face. Aneurysm is an abnormal dilatation of blood vessel wall, there are two types of aneurysms true and false. All three layers of vessel wall are involved in true aneurysm whereas pseudo-aneurysm involves only those layers that are lacerated $[1,2]$. Due to the small size and superficial location of facial vessels, these are most commonly transected than lacerated $[1,3]$. Pseudoaneurysm most commonly develops after trauma presenting as a painless swelling, on examination, there is a palpable thrill and an audible bruit [1]. Radiological imaging modalities that can be used to identify the post-traumatic lacerations forming pseudo-aneurysms of these vessels include ultrasound and CT angiography [1]. According to a study, ultrasound is a cost effective, real timing and quick method for diagnosis however CT angiography is the modality of choice for vascular injury [3].

Pseudo-aneurysms can be slow growing after the trauma blood leaks into the surrounding layers and resulting in hematoma formation that stops further bleed. Later the endothelium re-develops forming an aneurysmal sac. These aneurysms may rupture resulting in blood loss. Therefore the prompt identification and treatment of these lesions is crucial. The treatment options include compression, surgical resection, ligation of vessels involved arteriography with embolization and sclerotherapy $[1,2]$.

\section{Case report}

A young patient with known co-morbid presented to the emergency department with a tender, slow growing swelling on the left side of face. He gave a history of blunt trauma to the face during fighting 15 days back. On examination he was vitally stable however there was a diffuse swelling on the left side of the face, it was tender to touch and slight pulsations on palpation in the left submandibular region. The overlying skin was bruised, there was mild facial disfigurement. The patient was given initial symptomatic treatment and was sent for a CT scan to rule out the possible cause of these signs and symptoms.

Due to the abnormal pulsations and history of trauma a CT Angiogram was performed which showed a lobulated contrast filled out-pouching measuring $1.3 \times 0.9 \times 1.4 \mathrm{~cm}$ (APxTSxCC) arising from the main left facial artery consistent with a pseudo-aneurysm. Left submandibular gland appeared enlarged and swollen with adjacent fat streaking and overlying soft tissue swelling. Left masseter muscle was also swollen and enlarged. There was stranding and streaking of fat along the 
left alveolar process. Rest of the vessels were normal on the CT scan. Findings were consistent with post-traumatic pseudoaneurysm with adjacent post-traumatic changes shown in Figures 1,2.

Patient was urgently referred to the intervention radiology department for angioembolization. Unfortunately, the patient lost to follow up from our hospital due to financial constraints and went to another welfare based tertiary care hospital for angioembolization and further management. On follow up patient was successfully managed and the aneurysm was embolized his hemoglobin improved after treatment. His attendants were in favor of non-surgical approach due to cosmetic reasons.

\section{Discussion}

Aneurysms are abnormal out-pouching in the vessel wall that can be divided into true, false and dissecting [1]. A true aneurysm involves all three layers of the vessel wall and are most commonly witnessed, these develop due to weakness in the walls of vessel [2]. Whereas false or pseudo-aneurysms are mostly post-traumatic due to laceration of one or two layers of vessel. The basic pathophysiology behind these pseudoaneurysms is that after trauma blood leaks through the vessel walls until the pressure of the resulting hematoma is equal to the mean arterial pressure. When this happens artery stops bleeding and the hematoma starts forming an endothelial lining. Later the central part of the hematoma liquefies forming an endothelial lined cavity that communicates with the vessel wall. The endothelial lined cavity forms a pulsatile mass that grows in size eventually due to the pulsatile flow of blood into this same sac through the vessel [1-5]. The growth of the aneurysm depends on the perivascular structures, those surrounded by tough fascia are slow growing whereas those surrounded by lax soft tissues grow rapidly [2]. Amongst the causes of pseudo-aneurysm development is tangential lacerations due to penetrating, blunt, and post-surgical trauma and fractures.

Facial artery rarely develops pseudo-aneurysm due to its superficial course that leads to its complete transection rather than laceration. Facial artery aneurysm can only rarely develop aneurysm due to trauma, orthographic surgery, molar tooth extraction, radiation exposure, malignancy and infection $[1,5]$. These aneurysms classically present as a painful, pulsatile swelling although rarely may be non-pulsatile due to thrombin formation. Cohen helped to differentiating a pseudo-aneurysm from an AV fistula by defining their characteristics. An AV fistula has a continuous vibratory thrill whereas a pseudoaneurysm has a bruit only during systolic phase. Also, a pseudo-aneurysm expands and compresses adjacent vessels while an AV fistula doesn't [2]. Our patient presented with a painful pulsatile swelling and had a history of trauma, his examination was also consistent with a vascular etiology.
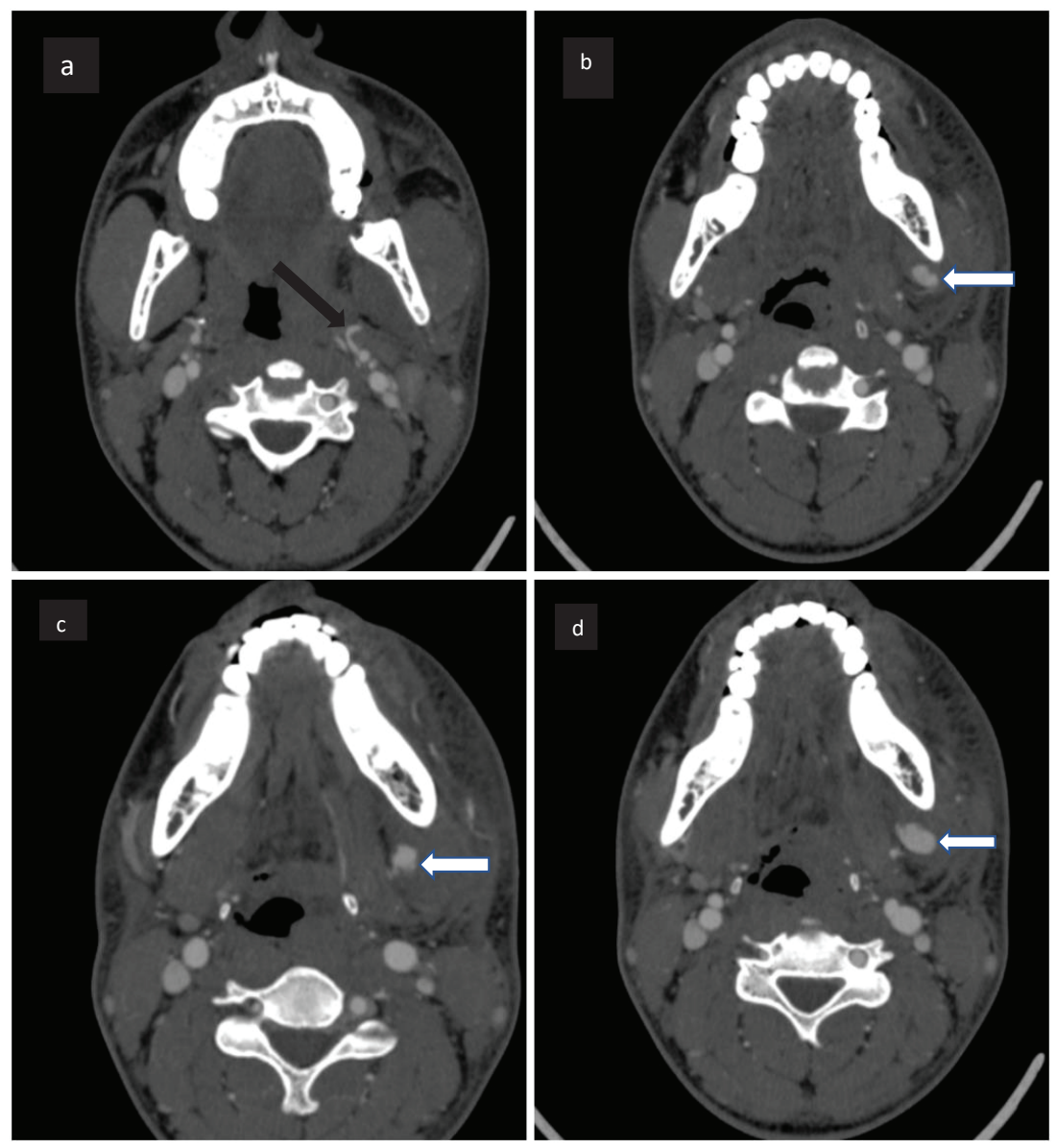

Figure 1: CT scan neck axial sections with contrast show Pseudoaneurysm (white arrow figure b, c and d) arising from left facial artery (black arrow figure a).

Citation: Altaf R, Ali I, Siraj R, lqbal J, Hassan F (2022) Post traumatic facial artery pseudoaneurysm-A case report. Glob J Medical Clin Case Rep 9(1): 001-003. DOI: https://dx.doi.org/10.17352/2455-5282.000145 

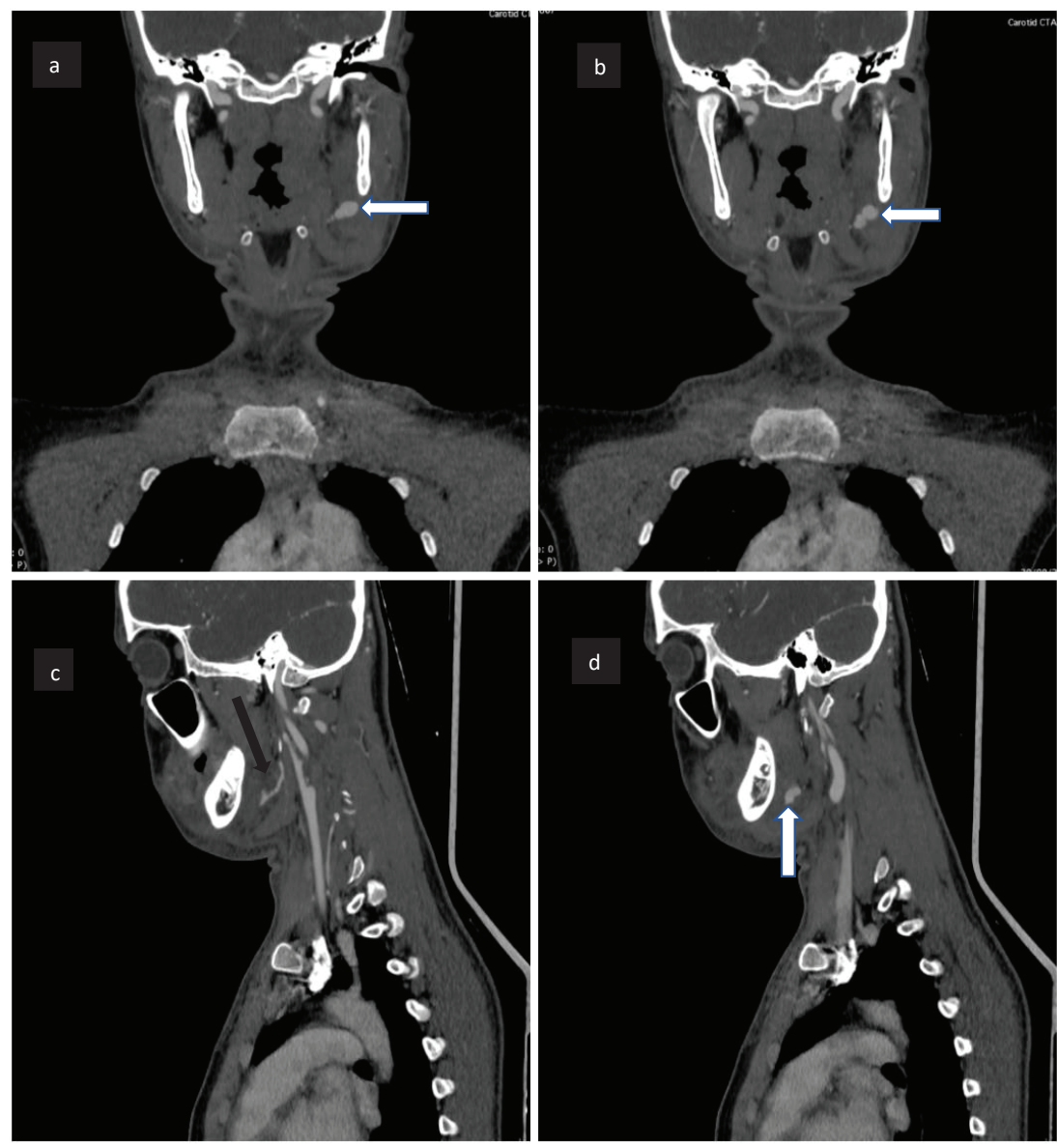

Figure 2: Ct scan neck cronal and sagital sections with contrast show pseudo-aneurysm (white arrow a, b and d) arising from left facial artery (black arrow figure c).

The diagnosis of these vascular lesions is important as hemorrhage can lead to severe complications and poor prognosis for the patient. Ultrasound is one of basic initial real time imaging modality used for the diagnosis of facial artery aneurysm as it is cost effective and radiation free. The imaging modality of choice however for these vascular lesions is a CT angiogram since 1980 [1-3]. CT Angiogram can highlight the minute anatomical details thereby making treatment easier $[2,3]$.

Treatment of facial artery aneurysms includes ligation, surgical excision and angioembolization. Of these angioembolization is the preferred treatment and should be attempted in all cases of pseudo-aneurysms. Other options should only be used if angioembolization is not available [15]. Our patient was also given the option of surgical treatment due to non-affordability patient went to a welfare hospital for embolization. Treatment however is mandatory to save patients from drastic complications.

\section{Conclusion}

Post-traumatic pseudoaneurysms of a facial artery are a rare presentation. However, the risk of bleeding associated with these vascular lesions makes it important for radiologists to correctly diagnose them. Radiologists should be careful and observant while reporting such cases to save the patients from severe bleeding.

\section{Ethical review}

This case report was written and images of the patient's CTA were added after approval from the patient. Patient's identity will be kept confidential.

\section{References}

1. Roy S, Jain N (2021) The Presentation and Management of Facial Artery Pseudoaneurysm: A Review of the Literature. Turk Arch Otorhinolaryngol 59: 76-79. Link: https://bit.ly/3pQ2eFa

2. Anand L, Sealey C (2017) Pseudoaneurysm of the facial artery following bilateral temporomandibular joint replacement: A case report. Oral and Maxillofacial Surgery Cases 3: 11-14. Link: https://bit.ly/3FWHSQA

3. Cooperband BR, Friedel W, Bhatt GM, Eisig S (1989) False aneurysm of the facial artery. J Oral Maxillofac Surg 47: 1327-1329. Link: https://bit.ly/3FUEw0f

4. Jenq KY, Panebianco NL, Lee PA, Chen EH, Dean AJ (2010) Diagnosis of a facial artery pseudoaneurysm using emergency bedside ultrasound. J Emerg Med 38: 642-644. Link: https://bit.ly/3HwfncM

5. Nakagawa K, Yasuda T, Kobayashi N, Urabe K (2020) Huge true aneurysm of the facial artery treated with internal trapping and surgical excision: a case report. J Surg Case Rep 2020: rjaa375. Link: https://bit.ly/3mR3fLt 